\title{
Una propuesta experimental en la enseñanza de la música en secundaria*
}

\author{
A PROPOSAL OF EXPERIMENTAL MUSIC IN SECONDARY TEACHING
}

UMA PROPOSTA DE MÚSICA EXPERIMENTAL NO ENSINO SECUNDÁRIO

\section{Ana María Botella Nicolás** José Rafael Adell Valero***}

Cuadernos de Música, Artes Visuales y Artes Escénicas

/ Volumen 11 - Número 1 / enero - junio de 2016

/ ISSN 1794-6670/ Bogotá, D.C., Colombia / pp. 67-81

Fecha de recepción: 15 de abril de 2015 | Fecha de aceptación: 26 de octubre de 2015 | Disponible en línea:

30 de mayo de 2016. Encuentre este artículo en http://

cuadernosmusicayartes.javeriana.edu.co/

doi:10.11144/Javeriana.mavae11-1.peem

* Artículo de investigación, fruto de un trabajo que lleva por título El desarrollo de las competencias musicales a través del aprendizaje cooperativo por proyectos en el aula de música de secundaria, desarrollado en el marco del Máster Universitario en Investigación en Didácticas Específicas de la Facultad de Magisterio de la Universitat de València (España).

**Doctora en Pedagogía, Licenciada en Musicología y maestra en Pedagogía Musical. Profesora del Departamento de Didáctica de la Expresión Musical, Plástica y Corporal, de la Facultad de Magisterio de la Universitat de València.

** Licenciado en Ciencias Biológicas. Profesor de música del Centro Florida Universitaria y del CEIP la Cañada de Valencia, y profesor superior de Tuba por el Conservatorio Superior de Música de Valencia. 


\section{Resumen}

En el presente artículo se describe una experiencia de enseñanza de la música en secundaria, aplicando el Método Cooperativo por Proyectos. Se trata de determinar su eficacia y pertinencia en el marco del aprendizaje por competencias mediante un proceso de investigación-acción que se llevó a cabo en Valencia (España) con alumnos de $2^{\circ}$ y $3^{\circ}$ de Enseñanza Secundaria Obligatoria (ESO). Para comprobar su efectividad se ha contrastado dicho método con otro de referencia: la Clase Magistral. Las conclusiones se justifican mediante un análisis estadístico, realizado de manera cualitativa y cuantitativa.

La propuesta se realiza con el fin de comprobar si esta experiencia da respuesta a las necesidades sociales que exige el mundo actual, y también si favorece que los estudiantes piensen y actúen de manera reflexiva, en colaboración con los demás y desde una perspectiva creativa y crítica, según los conceptos teóricos de Schafer, Delalande, Reibel y Paynter.

Palabras clave: música; trabajo por proyectos; aprendizaje cooperativo; secundaria

\section{Abstract}

This article is focused on the teaching of music in Secondary education, using the cooperativeproject method described in the experience. It is their effectiveness and relevance in the context of learning skills through a process of action research which was carried out in Valencia (Spain) with students of 2nd and 3rd of Secondary Education (ESO). To test their effectiveness has been proven that method with another of reference: the Master Class. The conclusions are justified by statistical analysis conducted qualitatively and quantitatively. The proposal is made in order to check whether this experience provides answers to social needs demanded by today's world and whether encoura- ges the students to think and act reflectively collaborating with others and acting from a creative and critical perspective, following Schafer theoretical concepts, Delalande, Reibel and Paynter.

Keywords: music; work projects; cooperation learning; secondary

\section{Resumo}

Neste artigo, focado no ensino da música no secundário, usando o método descrito por experiência cooperativa Project. É a sua eficácia e relevância no contexto de competências de aprendizagem através de um processo de pesquisa-ação que foi realizada em Valência (Espanha), com estudantes de $2^{\circ}$ e $3^{\circ}$ do Ensino Secundário (ESO). Para testar a sua eficácia tem sido provado que com outro método de referência: a classe principal. As conclusões são justificadas pela análise estatística realizada qualitativamente e quantitativamente.

A proposta é apresentada no fim de verificar se esta experiência fornece respostas às necessidades sociais exigidos pelo mundo de hoje e se incentiva os alunos a pensar e agir reflexivamente colaborar com os outros e agir a partir de uma perspectiva criativa e crítica, a seguir conceitos teóricos de Schafer, Delalande, Reibel e Painter.

Palavras chaves: música; projetos de trabalho; cooperação; secundário 


\section{INTRODUCCIÓN}

Para un profesional de la docencia de la Educación Artística, el enfoque didáctico supone un reto que debe afrontar sin prejuicios, con un espíritu abierto y con la disposición a aportar sugerencias y proyectos que ayuden al desarrollo renovado de la materia. Si además se trata concretamente de la enseñanza musical, dicho reto puede ser más amplio, puesto que las metodologías propuestas desde mediados del siglo XX por Schafer, Delalande, Reibel, Paynter y otros, sitúan el aprendizaje en parámetros inéditos y originales.

La puesta en práctica de sus teorías constituye una transformación fundamental en la enseñanza de la música. Sin embargo, a pesar de su importancia, no se les ha dedicado la atención que merecen, una razón más que suficiente para verificar su eficacia:

Los cambios en la educación constituyen un proceso lento. Las viejas convicciones se resisten hasta el final, y los profesores cuyo trabajo apunta en nuevas direcciones a menudo se encuentran en una minoría vulnerable. No obstante, de vez en cuando ocurre algo que aglutina los distintos esfuerzos a favor del cambio dándole a éste una aprobación formal. (Paynter, 1999, p. 10)

Así pues, esta investigación se construyó sobre los cimientos teóricos de los autores citados cuyo espíritu y conceptos han permanecido en la base metodológica del proceso.

Innovar nunca es sencillo, como tampoco lo es experimentar nuevas formas metodológicas que, aunque estén ya prácticamente en uso desde hace unos años, supongan un esfuerzo de adaptación adicional a la labor del profesor. Por tanto:

Frente a la pasividad, hay que recuperar el dinamismo propio del ser humano, favoreciendo en el alumno la búsqueda de lo nuevo, la creación y la ampliación de sus posibilidades. (Marina,

2010, p. 49)

Actitud que nos conduce hacia las aspiraciones educativas actuales que suponen una postura comprometida en la búsqueda de nuevas alternativas.

Emprender un proyecto conlleva un esfuerzo que se debe realizar si se considera necesario replantear el sistema de enseñanza, actualizarlo y ver si existen posibilidades de mejora para el mismo. Por otro lado, es importante concretar las experiencias y cotejar si las hipótesis pueden mejorarse después de hacer este ejercicio de confrontación y de estructuración.

\section{MARCO TEÓRICO}

Schafer se cuestiona: “¿Por qué enseñar música? ¿Qué debería enseñarse? ¿Cómo debería enseñarse? ¿Quién debería enseñarla?" (2008, p. 32). Todo ello nos lleva a la inevitable cuestión: ¿Qué queremos que aprendan nuestros alumnos? Debemos ser conscientes de que con los estudiantes de secundaria no se trata de hacer profesionales de la música, sino de desarrollar sensibilidades, capacitar en la práctica de experiencias transversales, disfrutar aprendiendo, cultivar la creatividad y, en suma, desarrollar la asignatura del modo más vivencial posible para despertar su interés. El autor también define la música como algo que suena: si no suena, no es música:

¿Cuáles son los ingredientes básicos de la música? ¿Cuáles son los prístinos elementos a partir de los que puede estructurarse, y cuáles son los potenciales expresivos que el grupo humano o el individuo poseen para realizar estos fines? (Schafer, 2008, p. 17) 
Según esto, el lenguaje musical sería como un vehículo que nos permite conseguir un fin y no considerarlo como el fin en sí mismo, lo cual nos aleja de las pizarras y los cuadernos que no suenan y nos acercan a un enfoque más vivencial de la asignatura.

Delalande propone:

Los que, como yo, prefieren agudizar la curiosidad y la invención dejando mucho tiempo abierto

el abanico de posibilidades musicales, evitarán justamente ese cierre de campo al que conduce una notación codificada. (1995, pp. 127-128)

La partitura muchas veces no es necesaria, y por tanto, invertir tiempo en el aprendizaje del código, o aún peor, centrar en el código el aprendizaje musical, es un error: "No siempre es necesario escribir la música. Las nueve décimas partes de las músicas del mundo se componen y se transmiten sin partitura" (Delalande, 1995, p. 128).

Es obvio que la cultura actual necesita otros planteamientos distintos y la construcción de otras alternativas que cambien los aprendizajes. Si la música es sonido y movimiento, hay que empezar desde estas bases y posponer la notación y su compleja enseñanza para cuando sea significativo su aprendizaje. Es decir, cuando los alumnos necesiten hacer uso del lenguaje musical para poder escribir lo que interpretan o componen.

En cuanto a la metodología, Hemsy de Gainza, en la introducción a la obra de Schafer señala:

El pedagogo musical de la segunda mitad del siglo veinte no enseña pedagogía, y ni siquiera enseña música, sino HACE música con sus alumnos asumiendo así tanto las satisfacciones como los riesgos de su libertad. (2008, p. 9)

Desde estos parámetros se comprende que el cambio en el concepto musical y en su metodología son determinantes.

\section{EL MÉTODO COOPERATIVO POR PROYECTOS}

¿Cómo llevar a cabo estos principios en el aula de manera ordenada, reproducible y eficaz? Habrá que buscar el modo de conseguir que los estudiantes desarrollen las competencias necesarias, atendiendo a los objetivos marcados por el currículum de música, sin perder la esencia de dichas teorías. Schafer argumenta que "Lo que se enseña probablemente importe menos que el espíritu con que se imparte y se recibe" (2008, p. 18).

Después de considerar varias posibilidades, se fusionaron dos métodos que hasta ahora se habían trabajado por separado: el Método Cooperativo y el Método por Proyectos, generando así un efecto sinérgico para conseguir mejores resultados educativos. De esta asociación nace el Método Cooperativo por Proyectos.

La cooperación consiste en trabajar juntos para alcanzar objetivos comunes. Según Torrego y Negro:

No es sólo una alternativa metodológica y potencialmente eficaz para enseñar, sino una estructura didáctica con capacidad para articular los procedimientos, las actitudes y los valores propios de una sociedad democrática que quiere reconocer y respetar la diversidad humana.

(2012, p. 15) 
El aprendizaje cooperativo se realiza en grupos reducidos en los que los estudiantes trabajan conjuntamente para conseguir objetivos individuales y colectivos. Por ello, más que una agrupación de personas, es un sistema que permite compartir el mismo espacio físico, reforzarse unos a otros, aumentando las capacidades críticas. Según Ovejero, mejora "la autoestima y el funcionamiento de las capacidades intelectuales" (1993, p. 387), mientras se fomenta la autoayuda y el aprendizaje significativo.

En el Método de Proyectos, según Katz, citado en Clark: "un proyecto consta de una investigación profundizada, realizada por niños, de un tema digno de su tiempo, atención y energía" (2006, p. 2). Esta definición un tanto simplificada deberá ampliarse para su mejor comprensión.

Tal y como indica Clark un proyecto incluye tres fases:

1. El alumnado junto a su maestro, eligen y discuten un tema a explorar.

2. El paso siguiente consiste en que los alumnos realizan investigaciones directas y después organizan y disponen sus hallazgos.

3. El proyecto culmina con una serie de preguntas y respuestas así como de la puesta en común de sus investigaciones. (2006, p. 2)

Los estudiantes investigan, individualmente o en grupo, lo que para ellos es más relevante, lo cual convierte este aprendizaje en significativo, no solo por la temática que eligen, sino también por las diversas fuentes de información de donde toman los datos: Internet, televisión, artículos de revistas... Finalmente, se pone todo el material en común y se construye el fundamento de la investigación para llegar así a resultados concretos.

Como se puede apreciar, hay muchas posibilidades si se unifican los dos métodos: en ambos casos se trata de trabajar de manera abierta, fomentando la curiosidad, la experimentación y el desarrollo de estrategias que lleven a la obtención de resultados óptimos. Piaget, (1964), citado en Siegel y Brainerd, comenta:

El principal objetivo de la educación es crear hombres que sean capaces de hacer cosas nuevas, no simplemente repetir lo que otras generaciones han hecho, hombres que sean creativos, inventivos, descubridores. El segundo objetivo es formar mentes que puedan ser críticas; que puedan verificar, y no aceptar todo lo que se les presenta (...) Necesitamos alumnos que sean activos, que aprendan temprano a investigar por sí mismos, en parte a través de su propia actividad espontánea, y en parte a través del material que les presentamos. (1978, pp. 170-171)

De esta manera, conjugando ambos métodos se aprecia que el todo es más que la suma de las partes y que, en ambos casos, se trata de fomentar el aprendizaje significativo, el trabajo entre iguales, la negociación permanente, la socialización de los alumnos, la necesidad de una organización, el descubrimiento, la planificación del trabajo, la diversidad... En este caso, el profesor juega un papel de espectador y/o guía que ayuda solamente en situaciones que parecen difíciles de solucionar.

\section{DISEÑO DE LA INVESTIGACIÓN}

La investigación-acción se ha realizado en un contexto real, con alumnos de secundaria: 26 de $2^{\circ}$ ESO (13 por grupo) y de 29 de $3^{\circ}$ (un grupo de 15 alumnos y otro de 14) en el Centro Escuela 2 de la Cañada en Valencia (España). Este centro se caracteriza por la utilización de 
metodologías alternativas y abiertas, así como por la búsqueda constante de nuevos enfoques pedagógicos.

Se ha optado por una estrategia metodológica de complementación, que conjuga un enfoque cuantitativo para describir las variables de interés y hacer las comparaciones necesarias, con un enfoque cualitativo para profundizar y completar la importancia de la información cuantitativa obtenida.

En el estudio de casos propuesto, y para contrastar la idoneidad del método, se formaron dos grupos de estudiantes distintos, a los que se impartieron los mismos contenidos, por una parte, a través de la metodología experimental y por otra, mediante la lección magistral, para comprobar cuáles habían sido los resultados obtenidos. Posteriormente se realizó un análisis cuantitativo y cualitativo que permitió constatar la validez del método propuesto.

Con objeto de conocer el nivel de conocimientos previos respecto al tema se pasó un pretest. Tras la intervención, se pasó un postest para constatar si se habían producido cambios significativos. No obstante, y dado que el test es un elemento cuantificable objetivo, hubo que añadir una encuesta de percepción a fin de recoger sus percepciones a lo largo del proceso, y permitir que valores como la cooperación, la implicación en el proyecto y otros elementos más subjetivos tuvieran cabida en su valoración final.

Para el desarrollo de ambas metodologías, es decir, la Clase Magistral y el Método Cooperativo por Proyectos se tomaron los siguientes temas de la programación oficial de Música de Secundaria: En $2^{\circ}$ de ESO se hizo hincapié en la identificación y descripción de las características del sonido -altura, duración, intensidad y timbre, valorando el silencio y el sonido como parte integral de la música. Mientras que en $3^{\circ}$ se procedió a reconocer y describir algunos elementos y formas de organización y estructuración musical —ritmo, melodía, textura, repetición, imitación, y variación en una obra.

Dado que la Lección Magistral se considera el método de referencia y es conocida por su uso reiterado a lo largo del proceso educativo, solo se enuncia su desarrollo sin detallar cada una de sus fases:

1. Exposición.

2. Memorización de lo expuesto.

3. Verbalización de lo memorizado (prueba oral o escrita).

4. Valoración del resultado.

La clase magistral es siempre individual y homogénea. Atiende principalmente a la generalización, lo común y lo predecible.

El desarrollo de la actividad con el Método Cooperativo por Proyectos se propuso de la siguiente manera:

1. Elección de una obra que facilite el trabajo de los aspectos que se desean desarrollar.

2. Formación de grupos con base en las voces de la obra.

3. Trabajo de cada voz con percusión corporal.

4. Interpretación con instrumentos no convencionales.

5. Formación de grupos de expertos para intensificar la coordinación y la cooperación mediante equipos de trabajo que actúen por consenso.

6. Realización del ensayo con todas las voces a la vez. 
1. Elección del tema. Como introducción se presentó una serie de videos en los que grupos de reconocidos percusionistas, como Mayumana y Stomp, hacen música con instrumentos no convencionales. Tras esta presentación se ofrecieron diversas alternativas que cubrían los contenidos que se deseaba trabajar. Finalmente por consenso general se tomó un acuerdo. De este modo, en $2^{\circ}$ de ESO se puso en práctica la obra de Silvia León Aire rítmico, diseñada para ser interpretada con abanicos. En $3^{\circ}$ se escogió la obra Balloonology de Jeremi Telfort escrita para globos.

2. La formación de grupos. Los estudiantes se organizaron en función de las voces que formaban el conjunto de la obra.

3. Se trabajó cada voz con percusión corporal. Cada voz, independientemente, descifró la partitura con la ayuda del profesor. Se realizó una interpretación sin instrumentos usando únicamente el propio cuerpo como elemento sonoro.

4. Elección de los "instrumentos musicales". Como ya se ha indicado, se trabajó con abanicos y con globos, a fin de estimular la escucha de sonidos poco habituales que despertaran su atención y sensibilizaran hacia otros desconocidos.

5. Se formaron grupos de expertos para intensificar la coordinación, y la cooperación mediante equipos de trabajo que actúen por consenso. Se crearon dichos grupos en los que se hizo especial hincapié en la integración de las voces, de manera que el silencio, las entradas, las esperas y otros elementos de la composición se fueran respetando y aprendiendo.

6. Ensayo de todas las voces a la vez. Tras el trabajo de coordinación de los grupos de expertos, se reestablecieron los grupos originales y se puso todo en común.

\section{DESARROLLO DE LA ACTIVIDAD}

En la presentación de la actividad se hizo hincapié en que, al margen del resultado final que por supuesto sería realmente interesante-, había que insistir en el proceso de ejecución. Se trataba de realizar un trabajo reflexivo en el que se buscaba la implicación y motivación de todos los estudiantes.

Cada uno, pues, tenía que aportar su experiencia y también había que consensuar acuerdos y decisiones diversas en las que el profesor participaba nada más que como un guía o apoyo en momentos de dificultad.

En esta situación se descubre que se puede hacer música de muchas maneras, que siempre que exista un orden dentro de la variedad y la necesidad de expresar algo, el fenómeno sonoro tendrá una importancia fundamental. Además, este aprendizaje debe conseguir que los estudiantes se interesen y respeten cualquier forma musical, sea cual sea su procedencia o estilo.

En realidad se trataba de poner el énfasis en el proceso de creación, anteponiéndolo incluso a los resultados que se conseguirían en él. Surgieron improvisaciones, cambios en la partitura y variaciones de todo tipo fruto de su interés. Llegar a un consenso que podía actuar incluso en contra de opiniones individuales fue muy interesante, porque aunque por supuesto se experimentaron sugerencias personales, algunas tuvieron que desestimarse porque obstaculizaban el efecto del conjunto.

El objetivo consistió en generar un orden, dentro de unas características específicas que, además de educar, ayude a liberar tensiones y a darles un cauce adecuado para su aprovechamiento y expansión. El trabajo diseñado para que se realizara con diferentes grupos de alumnos fue uno de los elementos socializadores más importante de la acción. 


\section{LAS SESIONES}

En la primera sesión, se informó al alumnado de las características de la experiencia que iban a realizar, destacando que era un trabajo cooperativo en el cual iban a intervenir todos, cada uno con un rol distinto dentro del grupo que, más tarde, se pondría en común para conseguir un efecto musical determinado. Por supuesto se iban a formar tantos grupos como voces diferentes, que finalmente unirían su trabajo, con lo cual no se eximía a nadie de su participación ni de su responsabilidad, según su propia experiencia musical.

Tras el proceso de elección de la obra, mencionado con anterioridad, se pasó el pretest para determinar el nivel de conocimientos previos sobre el tema. Se presentó la partitura y se repartió entre los estudiantes.

La sesión terminó con la división de la clase en cuatro grupos en $2^{\circ}$ y cinco en $3^{\circ}$. Para la realización de estas agrupaciones fue necesaria la intervención del profesor, que desestimó la elección de los estudiantes que se habían agrupado siguiendo criterios de simpatía y amistad. Por el contrario, el criterio escogido para la formación de los equipos fue que cada grupo estuviera compensado respecto a conocimientos musicales y hábitos de trabajo.

En la segunda sesión se inició la tarea independiente de cada grupo. Tanto en $2^{\circ}$ como en $3^{\circ}$ en primer lugar se realizó una actividad de percusión corporal durante media sesión, basada en la partitura. Los alumnos tienen nociones de lectura de lenguaje musical, pero no son capaces de descifrar correctamente una partitura al 100\%. Posteriormente se inició una exploración de las posibilidades sonoras de los instrumentos (abanicos y globos) que se iban a utilizar. De forma natural se produce una experimentación lúdica y espontánea al contacto con los cuerpos sonoros.

Uno de los problemas que se presentó al principio fue el de discriminar la calidad y reproducibilidad de los determinados sonidos, o diferenciar si eran agudos o graves. De ello dependía el efecto de las diferentes texturas. De esta manera se impuso un conocimiento y estudio de los diferentes timbres que se pueden obtener: cerrar, abrir, golpear, rascar, soplar...

Respecto a la unidad didáctica o propuesta de trabajo relativa a un proceso de enseñanzaaprendizaje que se pretendía desarrollar —en este caso, las características del sonido en $2^{\circ}$ y elementos musicales básicos en $3^{\circ}$ - nada se dejó al azar, aunque hubo momentos en que se plantearon modificaciones respecto al proyecto inicial, dado que los contextos en los cuales se desarrolló la acción necesitaron hacer alguna adaptación imprevista. El azar a veces juega un importante papel, y puede acentuar aspectos lúdicos nada desdeñables. En realidad, aunque no se planteó como un juego, como se fue viendo, este aspecto tuvo una gran importancia en la creación de un ambiente distendido. Nunca se debe dejar de lado el "para qué se educa" y a este respecto el resultado de la actividad fue completamente satisfactorio porque intervinieron en él aspectos imposibles de desarrollar mediante métodos tradicionales.

Poco a poco fueron tomando conciencia de que esta experiencia no tiene nada que ver con la rutina y que se les iba a exigir una actitud y un nivel más activo. Verdaderamente el problema consiste en que para coordinarse aparecen opiniones divergentes que hay que solucionar mediante el análisis y la reflexión personal, renunciando a veces a sus propias ideas. Pero, progresivamente, se van dando cuenta de que el éxito de sus experiencias depende de ellos mismos y eso les hace ser responsables de sus actuaciones.

La tercera sesión se inició con la revisión del trabajo desarrollado anteriormente. A continuación se crearon grupos de "expertos" formados por alumnos que actuaban como 
representantes de cada una de las voces. Su misión consistió en explicarse unos a otros cómo eran las voces que habían trabajado, generando así un conocimiento global de la obra.

Siempre hay alumnos que son más creativos e impulsivos pero, en conjunto, a medida que la experiencia se desarrollaba se iba consiguiendo aumentar la apertura a la percepción y la autoconfianza, la agilidad y la persistencia para conseguir los logros propuestos, la concentración y la actitud proactiva durante el desarrollo de las sesiones. Y también fue notable el grado de participación que se iba produciendo.

Al trabajar conjuntamente, se iban percatando de que se puede hacer música de muchas maneras siempre que se produzca un orden dentro de la variedad y una necesidad por expresar algo nuevo. Los debates que se originaron en esta sesión demostraron que iban consiguiendo un grado de madurez superior al de los comienzos.

Es natural que al principio se produzca algún atisbo de desconcierto, puesto que al faltar la autoridad indiscutible del profesor, presente en la Lección Magistral, y al depender todo de ellos mismos, se enfrentan a una situación poco habitual que les sitúa en la coyuntura de tener que escucharse, respetarse y valorar cada aportación por muy extraña que parezca. Por supuesto debe haber un clima de comprensión absoluto y unas ganas de responsabilizarse de un trabajo que, aunque se desconozca el resultado, implica a todos en el mismo.

Durante la cuarta sesión, se procedió a un ensayo por grupos. La sorpresa ante los resultados que se iban obteniendo fue un estímulo muy importante para ellos. Cada grupo actuó independientemente mostrando sus logros. Además, en este punto los estudiantes empezaron a distinguir claramente las nociones teóricas que se pretendía desarrollar y comenzaron a entender la obra así como sus posibilidades expresivas. Se va estableciendo paulatinamente un compromiso entre adaptar la partitura a nuestra forma de interpretación y los requerimientos que ella sugiere: los silencios, las entradas, y las distintas actuaciones que hay que respetar, se fueron incorporando al trabajo, consiguiendo, finalmente, que el resultado fuera comprensible.

Se puso de manifiesto el rendimiento conseguido por cada uno de los grupos así como la implicación personal de sus componentes. El profesor tuvo que alentar a los estudiantes menos implicados, intentando que participaran de manera activa y procurando que su pasividad o miedo al ridículo desaparecieran.

Llegados a la quinta sesión, se realizó un ensayo general en el que se puso en común el trabajo de cada grupo. En este momento, se tuvo que corregir alguna forma de participación defectuosa que afectara al conjunto de la obra. Mediante este procedimiento los estudiantes deben comprender la importancia de la interacción y de la participación activa individual que se debía incorporar absolutamente al efecto total de los resultados. También deben haber asumido la adquisición de una seguridad en el planteamiento y en la determinación que finalice con un resultado satisfactorio.

Finalmente se pasó el postest con objeto de estimar el incremento de la adquisición de contenidos. Posteriormente se realizó la encuesta de percepción con objeto de recoger las valoraciones y sensaciones subjetivas del proceso vivido por el alumnado.

Si la experiencia resultó novedosa para ellos, aún lo fue más descubrir el grado de conocimientos musicales inesperados que habían adquirido. Además, según las propias palabras de los estudiantes, esos conocimientos se habían fijado de manera mucho más permanente que impartidos según el proceso de la Lección Magistral. 


\section{ANÁLISIS DE LOS DATOS Y DESCRIPCIÓN DE LOS RESULTADOS}

Se utilizó el programa IBM SPSS Stadistics versión 12 como técnica para analizar los datos obtenidos mediante la aplicación de los correspondientes tests estadísticos:

- Prueba de Kolmogorov-Smirnov con objeto de comprobar que las muestras se ajustan a una distribución normal o gausiana.

- Test t-student para muestras independientes con el fin de demostrar que no existen diferencias significativas entre ambos grupos antes de la intervención.

- Test ANCOVA para la comparación de ambas metodologías.

Los resultados obtenidos para $2^{\circ}$ y $3^{\circ}$ de ESO se pueden observar en la tabla y gráficos 1 y 2 respectivamente. Previo al análisis de los datos, se realizaron unas comprobaciones estadísticas con objeto de determinar las siguientes cuestiones:

1. Los grupos creados para cada metodología (Lección Magistral y Método Cooperativo por Proyectos) parten de niveles de conocimientos similares. Para ello se pasaron sendas pruebas $K-S$ con objeto de confirmar que los datos se ajustaban a una distribución normal o gausiana. Efectuada esta comprobación, se pasó la prueba t-student para muestras independientes con la hipótesis nula HO: No existe diferencias significativas entre los resultados del pretest en ambos grupos. Los resultados del test no permitieron descartar la hipótesis nula, por lo tanto, se asume que los grupos de cada metodología parten de niveles similares de conocimientos.

2. Comparación de ambas metodologías empleadas así como la influencia del pretest sobre el postest. Para ello se realizó un test estadístico ANCOVA cuyos resultados para $2^{\circ}$ y $3^{\circ}$ fueron: Que el pretest estaba afectando a los resultados del postest, es decir, los niveles de conocimientos iniciales afectan a los niveles de conocimientos tras la intervención. Por otro lado, respecto a la existencia de diferencias entre las dos metodologías, el test indica que no se puede rechazar la $\mathrm{HO}$ y por tanto, se concluye que no hay diferencias significativas entre una y otra.

La comparación entre los resultados del pretest y el postest ponen en evidencia que en ambas metodologías los estudiantes han mejorado tras la intervención. En $2^{\circ}$ de ESO la diferencia entre el pretest y el postest para la metodología tradicional es de 1,6 puntos (sobre 10), mientras que en el aprendizaje cooperativo por proyectos es de 1,1 puntos, tal y como se puede observar en la tabla 1:

Tabla 1. Comparación de resultados obtenidos en ambas metodologías en $2^{\circ}$ ESO

\begin{tabular}{ccccc}
\hline & \multicolumn{2}{c}{$\mathbf{2}^{\circ}$ ESO } \\
\hline Metodología & \multicolumn{2}{c}{ Clase magistral } & \multicolumn{2}{c}{ Por proyectos } \\
Test & Pretest & Postest & Pretest & Postest \\
Media & 6,7 & 8,3 & 6,6 & 7,7 \\
\hline
\end{tabular}




\section{Comparación de metodologías $2^{\circ}$ ESO}

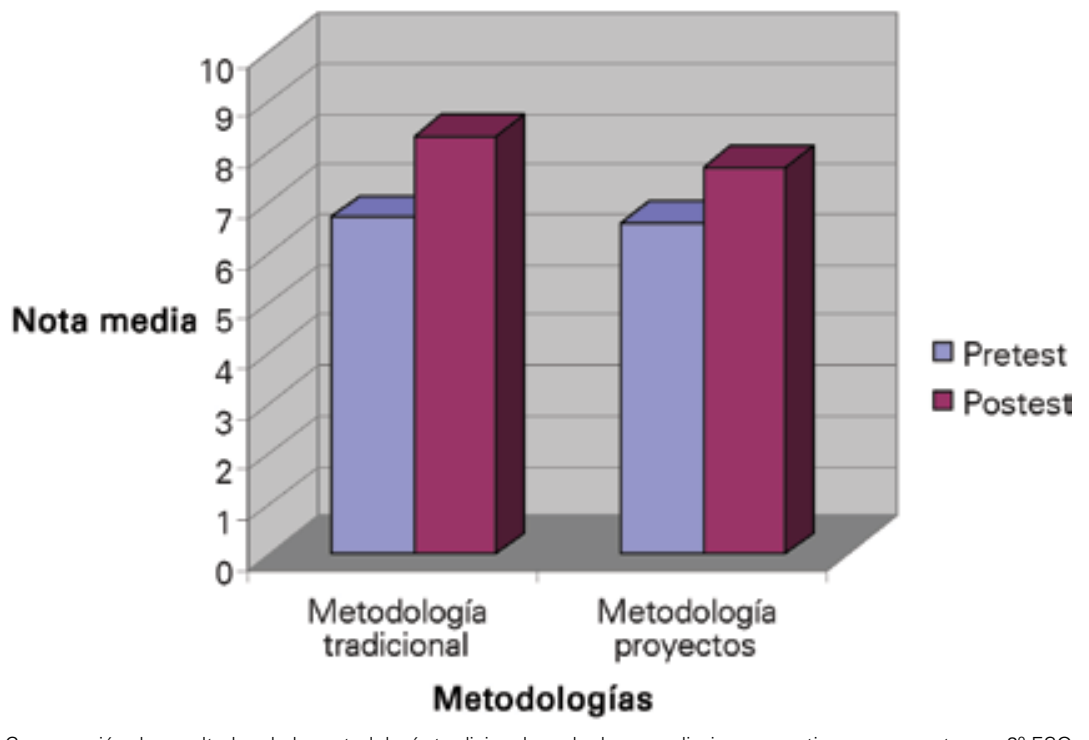

Gráfico 1. Comparación de resultados de la metodología tradicional con la de aprendizaje cooperativo por proyectos en $2^{\circ}$ ESO.

En $3^{\circ}$ la diferencia entre el pretest y el postest en la metodología tradicional es de 1,9 puntos, mientras que en el caso del aprendizaje cooperativo por proyectos es de 1,7 puntos. En principio se podría pensar que da mejores resultados la clase magistral que el método cooperativo, pero como se ha demostrado con el test ANCOVA las diferencias no son significativas:

Tabla 2. Comparación de resultados obtenidos en ambas metodologías en $3^{\circ}$ ESO

\begin{tabular}{ccccc}
\hline & \multicolumn{3}{c}{$\mathbf{3}^{\circ}$ ESO } \\
\hline Metodología & \multicolumn{2}{c}{ Clase magistral } & \multicolumn{2}{c}{ Por proyectos } \\
Test & Pretest & Postest & Pretest & Postest \\
Media & 5,7 & 7,6 & 6,1 & 7,8 \\
\hline
\end{tabular}

\section{Comparación de metodologías $3^{\circ}$ ESO}

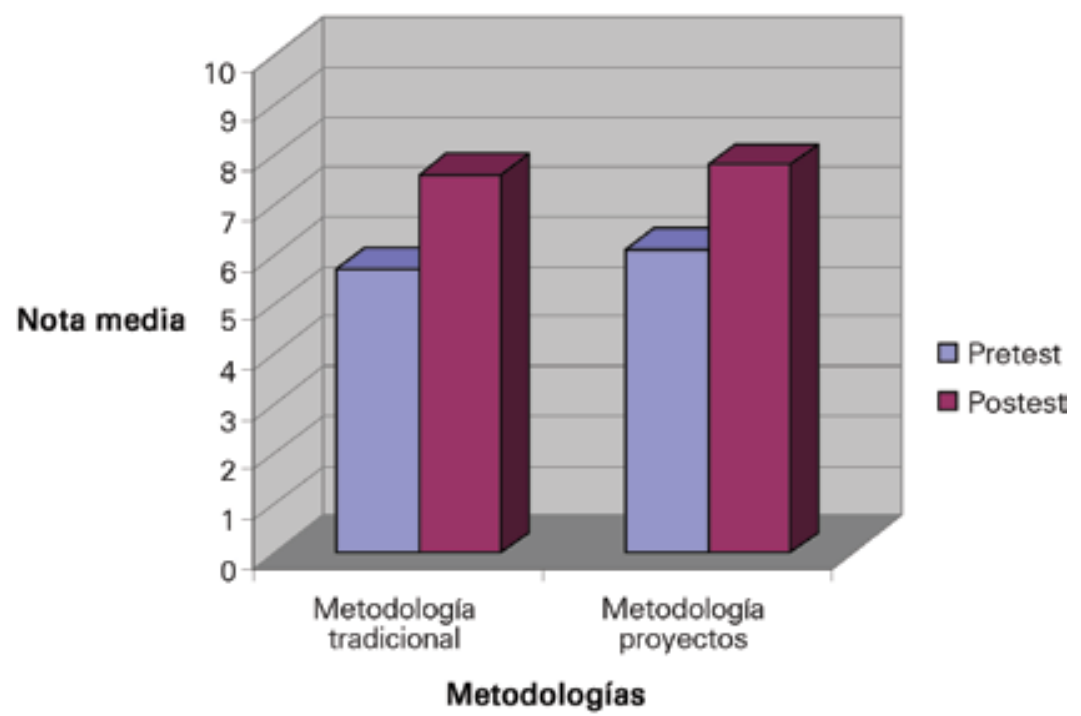

Gráfico 2. Comparación de resultados de la metodología tradicional con la de aprendizaje cooperativo por proyectos en $3^{\circ}$ ESO. 
Es importante señalar que se ha utilizado el criterio de prudencia valorativa en lo que respecta a la evaluación de la experiencia. Esto significa que la prueba escogida como herramienta de evaluación está claramente enfocada a la metodología de la clase magistral. Lo que se pretende con esta medida es demostrar que incluso en condiciones evaluatorias desfavorables, el método cooperativo por proyectos es capaz de dar resultados del mismo nivel de la clase magistral. Con ello está demostrando ser muy eficaz, ya que sus verdaderas potencialidades no se están estimando; solo se valora uno de sus aspectos que es la adquisición de conocimientos.

Respecto a los resultados de la encuesta de percepción que realizaron los estudiantes de $2^{\circ}$ y $3^{\circ}$ de ESO, se pueden observar en las tablas 3 y 4 respectivamente:

Tabla 3. Comparación de los resultados de la encuesta de percepción en $2^{\circ}$ ESO.

\begin{tabular}{ccc}
\hline & $2^{\circ}$ ESO & \\
\hline Metodología & Clase magistral & Por proyectos \\
Media & 3,9 & 4,5 \\
\hline
\end{tabular}

Tabla 4. Comparación de los resultados de la encuesta de percepción en $3^{\circ}$ ESO.

\begin{tabular}{ccc} 
& & $3^{\circ}$ ESO \\
\hline Metodología & Clase magistral & Por proyectos \\
Media & 3,6 & 4,6 \\
\hline
\end{tabular}

Tal y como se puede constatar en los gráficos 3 y 4, las puntuaciones son significativamente mejores en el caso de la Metodología por Proyectos:

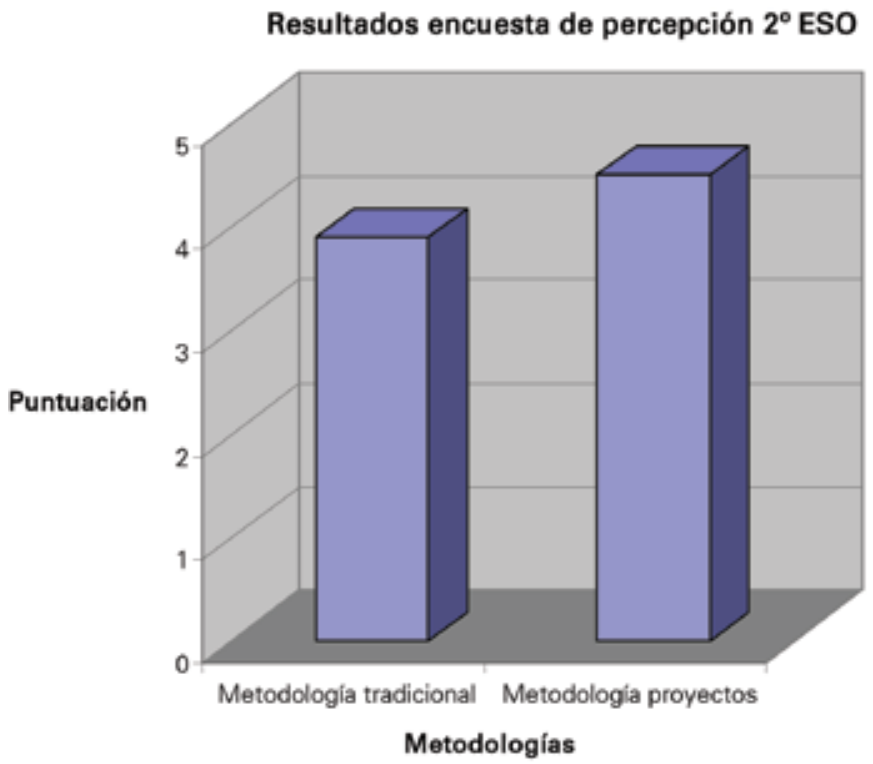

Gráfico 3. Comparación en las puntuaciones obtenidas en la encuesta de percepción $2^{\circ}$ ESO.
Resultados encuesta de percepción $3^{\circ}$ ESO

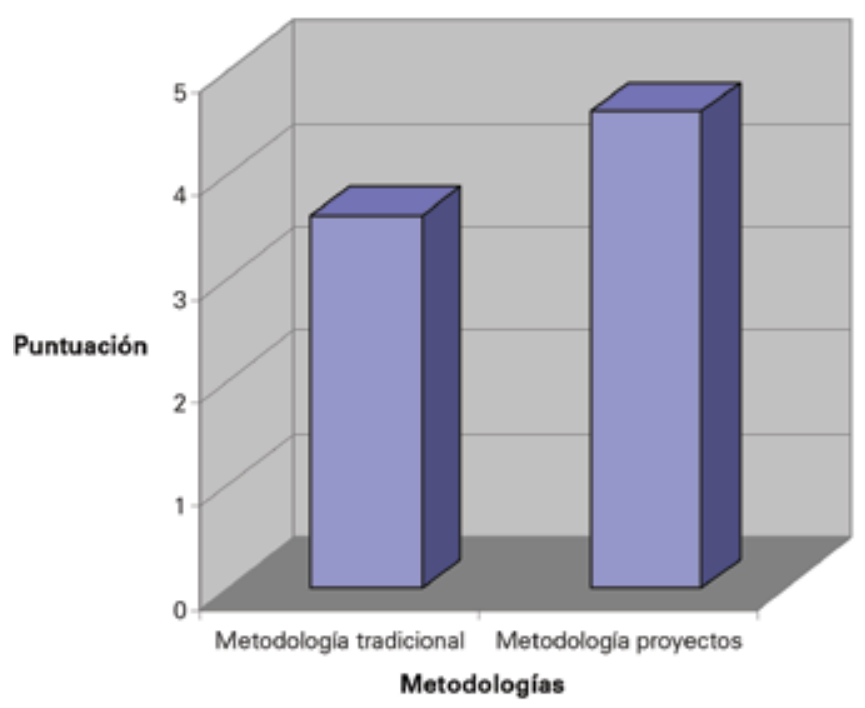

Gráfico 4. Comparación en las puntuaciones obtenidas en la encuesta de percepción $3^{\circ} \mathrm{ESO}$ 
La diferencia de puntuación que se da en $2^{\circ}$ es de 0,6 puntos y en el caso de $3^{\circ}$ es de 1 punto. Hay que señalar que dichos resultados están referenciados en una escala Likert (de 1 a 5).

\section{CONCLUSIONES}

¿Dónde reside la ventaja de una metodología respecto a otra? El principal inconveniente de la lección magistral es que los alumnos aprenden los contenidos de manera puntual y descontextualizada, con lo que terminan por memorizar, para repetir la respuesta cuando surge la exigencia de la pregunta. Esto lleva al consabido problema de que los alumnos estudian solamente los días antes del examen a fin de superar la prueba. Se trata de un comportamiento lógico porque la utilidad de los contenidos trabajados se reduce al paso del control, y al finalizar este se olvidan tan rápidamente como se han adquirido, porque ya han cumplido su función. Además, este aprendizaje no supone una experiencia activa para ellos. De este modo no se construye, ni se relaciona, ni se hace propio el conocimiento.

La diferencia de trabajar por proyectos radica en que con esta metodología se posibilita un mejor aprendizaje por competencias, es decir, prepara a los alumnos para ser capaces de aplicar los conocimientos a situaciones concretas y novedosas, apartándose del modelo memorístico que se adquiere con el aprendizaje en la clase magistral. Con el aprendizaje cooperativo por proyectos se desarrollan una serie de habilidades o capacidades que se derivan del trabajo en grupo, tales como el respeto a la opinión de los demás, la convivencia, la negociación y la necesidad de tomar decisiones de manera consensuada.

Por otra parte, realizar un trabajo sobre un caso real hace que el aprendizaje sea significativo, permitiendo al alumno sumergirse en un proceso de metacognición que le hará ir construyendo su propio conocimiento a partir del trabajo que va desarrollando. Trabajando por proyectos se asegura la transferibilidad de los conocimientos a situaciones novedosas inesperadas y cambiantes, por lo que se capacita al alumno para encarar una solución satisfactoria que afronte y resuelva situaciones nuevas, de elementos no previsibles.

En cuanto a la experiencia como docentes, el grado de atención a la diversidad que se genera en el caso de la metodología por proyectos es significativamente mayor que en el caso contrario, ya que al trabajar en grupos y tener diferentes roles dentro de los mismos se favorece la posibilidad de incluir a todos en el trabajo.

El aprendizaje de contenidos conceptuales se genera mediante las dos metodologías ensayadas; pero existen grandes diferencias que se observan en la tabla 5 :

Tabla 5. Comparativa entre ambas metodologías.

\begin{tabular}{|c|c|c|}
\hline & Lección Magistral & $\begin{array}{c}\text { Aprendizaje cooperativo } \\
\text { por proyectos }\end{array}$ \\
\hline Rol del profesor & Fuente de conocimiento & Guía en el proceso de metacognición \\
\hline Adquisición de Conocimientos & $\begin{array}{l}\text { A través de una explicación y } \\
\text { posterior memorización }\end{array}$ & $\begin{array}{l}\text { A través de una experiencia, se } \\
\text { construye conocimiento }\end{array}$ \\
\hline $\begin{array}{l}\text { Permanencia de conocimientos } \\
\text { adquiridos }\end{array}$ & A corto plazo y sin proyección & $\begin{array}{l}\text { A largo plazo. Posibilidad de } \\
\text { transferencia a situaciones diferentes }\end{array}$ \\
\hline $\begin{array}{c}\text { Adquisición de competencias } \\
\text { básicas }\end{array}$ & Limitada y poco significativa & Amplia y socializadora \\
\hline Percepción de los estudiantes & $\begin{array}{l}\text { Positiva. Pero poco vivencial. Son } \\
\text { público. }\end{array}$ & $\begin{array}{l}\text { Muy positiva. Vivencial. Son } \\
\text { protagonistas. }\end{array}$ \\
\hline
\end{tabular}


Lo anterior permite concluir que la metodología de aprendizaje cooperativo por proyectos es capaz de cumplir con las exigencias del currículum en lo referente a contenidos conceptuales, y a la vez aportar un valor añadido en cuanto al desarrollo personal y social de los alumnos. Esto es posible porque, mediante esta metodología, se facilita el trabajo por competencias así como la atención a la diversidad, el pensamiento crítico y la capacidad de toma de decisiones.

Cabe destacar también que los tiempos empleados en ambas metodologías son distintos, puesto que difieren también los enfoques y los modos de aprendizaje. En la experiencia que se presenta en este trabajo se han tratado de equiparar los tiempos en ambos casos (la metodología por proyectos solo dispone de una sesión más que la clase magistral) porque se pretende hacer una comparativa con condiciones más o menos semejantes. Pero en la dinámica cotidiana de la clase se hace evidente que la metodología cooperativa por proyectos requiere más tiempo, dado que los aprendizajes son más completos y profundos y atañen no solo a los contenidos sino también a las competencias básicas.

Por otro lado, la motivación de los alumnos se incrementa notablemente al ser parte implicada en su aprendizaje. La responsabilidad de preparar un producto final que posteriormente será presentado ante la comunidad escolar y en ocasiones en otros países, les hace más responsables del trabajo a desarrollar. Su actitud mejora también frente al mismo. El nivel de conflictos generados en el aula se reduce significativamente al trabajar de este modo, así como el nivel de ausentismo frente a la asignatura. La percepción de los alumnos es que resulta fácil y agradable cursar la música, lo cual da relevancia a la materia y la hace situarse en el lugar que merece dentro del currículum escolar.

Si se necesita profundizar en las aspiraciones modernas que influyen en el sistema escolar y en las prácticas pedagógicas para llevar a buen fin el proyecto educativo, se tendrá que elegir un sistema de educación que contemple el "para qué se educa." Si se educa para la vida, no se puede dejar nuestra labor reducida a generar aprendizajes memorísticos superficiales y faltos de aplicación para los alumnos. Se ha de buscar formar personas críticas y capaces de enfrentar las diferentes situaciones que se van presentando a lo largo de la vida; la metodología cooperativa por proyectos constituye una herramienta para lograr este fin. El profesor explica a partir de las dificultades que van surgiendo; por eso es aprendizaje significativo.

Cabe destacar que en este modelo es posible integrar en el trabajo cotidiano a estudiantes de educación especial, fomentando su inclusión en el aula ordinaria. Precisamente en este caso concreto se han incorporado en cada curso dos alumnos con necesidades educativas especiales al proyecto desarrollado. Este es un tema que abre las puertas a nuevas expectativas de indagación y a posibles trabajos de investigación posteriores.

Por último, se puede afirmar que se ha vivenciado una experiencia de metodología musical que puede proponerse como una forma de interpretación y de creación, así como una valoración del proceso frente al resultado. En última instancia se trata de generar un armazón contundente respecto a la práctica musical, el trabajo por proyectos y la cooperación. Si esta actuación puede parecer obvia para profesionales de la música, no lo fue para alumnos que no tienen la posibilidad de interpretar una obra conjuntamente, y resultó un descubrimiento para ellos explorar una didáctica aplicada de modo real que además de enriquecerles les hizo comprender mecanismos desconocidos hasta entonces. Por otra parte, actuar con 
instrumentos no convencionales les sedujo, ya que al no estar viciados por otras escuchas, todo les resultaba original y gratificante.

El reto de emprender un proyecto semejante justifica con creces el esfuerzo realizado, el cual siempre será mejorable a medida que la experiencia se vaya afirmando y practicando.

\section{REFERENCIAS}

Clark, Ann-Marie. "Changing classroom practice to include the project approach". Early Childhood Research \& Practice. ECRP, Vol. 8, (2006): 1-11.

Delalande, François. La música es un juego de niños. Buenos Aires: Ricordi Americana, 1995.

Hemsy de Gainza, Violeta. "Prólogo", en Schafer, Murray. El rinoceronte en el aula. Buenos Aires: Melos, 2008.

Marina, José Antonio. "La competencia de emprender." Revista de Educación, 351, (2010): 49-71.

Ovejero, Anastasio. "El aprendizaje cooperativo: Una eficaz aportación de la psicología social a la escuela del siglo XXI". Psicothema, 5, (1993): 373-391.

Paynter, John. Sonido y estructura. Madrid: Akal, 1999.

Reibel, Emmanuel. L'écriture de la critique musicale au temps de Berlioz. Honoré Champion, 2005.

Schafer, Murray. El rinoceronte en el aula. Buenos Aires: Melos, 2008.

Siegel, Linda. S. y Brainerd, Charles J. Alternatives to Piaget: Critical essays on the theory. New York: Academic Press, 1978.

Torrego, Juan Carlos y Negro, Andrés, comps. Aprendizaje cooperativo en las aulas. Fundamentos y recursos para su implantación. Madrid: Alianza, 2012.

\section{Cómo citar este artículo:}

Botella, Ana María y Adell, José Rafael. "Una propuesta experimental en la enseñanza de la música en secundaria". Cuadernos de Música, Artes Visuales y Artes Escénicas, 11(1), 67-81, 2016. http://dx.doi.org/10.11144/Javeriana.mavae11-1. peem 
